\title{
BMP7 expression in the adult rat brain
}

\author{
Yuya Kusakawa ${ }^{a, b}$, Sumiko Mikawa ${ }^{a}$, Kohji Sato ${ }^{a, *}$ \\ a Department of Tissue E Organ Anatomy, Hamamatsu University School of Medicine, 1-20-1 Handayama, Higashiku, Hamamatsu, Shizuoka 431-3192, \\ Japan \\ ${ }^{\mathrm{b}}$ AICHI Medical College for Physical and Occupational Therapy, 519 Ichiba, Kiyosu, Aichi 452-0931, Japan
}

\section{A R T I C L E I N F O}

\section{Article history:}

Received 2 February 2017

Received in revised form 22 June 2017

Accepted 24 June 2017

\section{Keywords:}

Astrocyte

Neuron

Immunohistochemistry

\begin{abstract}
A B S T R A C T
Bone morphogenetic protein-7 (BMP7), a member of the transforming growth factor- $\beta$ (TGF- $\beta$ ) superfamily, has various effects in many biological events. However, there is little information on BMP7 expression in the adult central nervous system (CNS). Therefore, we investigated BMP7 levels in the adult rat CNS using immunohistochemistry. Abundant BMP7 expression was seen in astrocytes throughout the CNS and strong BMP7 expression was also observed in neuropils of the gray matter. Furthermore, BMP7 expression was observed in several kinds of neurons, including oxytocin, dopaminergic and noradrenergic neurons. These data suggest that BMP7 is widely expressed throughout the adult CNS, and support the idea that BMP7 plays pivotal roles in the adult brain, as well as in the developing brain.
\end{abstract}

(C) 2017 The Authors. Published by Elsevier Limited on behalf of International Brain Research Organization. This is an open access article under the CC BY-NC-ND license

(http://creativecommons.org/licenses/by-nc-nd/4.0/).

\section{Introduction}

Bone morphogenetic proteins (BMPs) are multifunctional cytokines involved in the development and differentiation of various tissues, including mesoderm induction and nerve formation and apoptosis (Bragdon et al., 2011). To date, about 20 BMPs have been identified and they form the largest group in the TGF- $\beta$ family. BMP receptors are present in the cell membrane as two types of serine/threonine kinase receptors (types I and II) that are essential for transmission of BMP signals (Bragdon et al., 2011). Different combinations of type I and type II receptors have different affinities for BMP ligands (Bragdon et al., 2011). Activation of type I receptors by type II receptors leads to phosphorylation of receptoractivated Smads (R-Smads; Smad1/5/8), and the phosphorylated $\mathrm{R}-\mathrm{Smads}$ bind to the common-mediator Smad (Co-Smad; Smad4) (Moustakas and Heldin, 2009). The Smad complexes are transported into the nucleus and regulate the expression of BMP target genes. BMPs also activate Smad-independent signaling pathways, such as the MAP kinase pathway (Massague, 2003), and BMP signals

Abbreviations: BMP, bone morphogenetic protein; BMPR, bone morphogenetic protein receptor; BSA, bovine serum albumin; CNS, central nervous system; CSPGs, chondroitin sulfate proteoglycans; GFAP, glial fibrillary acidic protein; IHC, immunohistochemistry; IR, immunoreactivity; PB, phosphate buffer; RT, room temperature; SVZ, subventricular zone; TGF- $\beta$, transforming growth factor $\beta$; TTBS, Tris-buffered saline containing $0.05 \%$ Tween-20.

* Corresponding author.

E-mail address: ksato@hama-med.ac.jp (K. Sato). are inhibited by secreted proteins that bind to BMPs, such as noggin, chordin, and neurogenesin-1 (Cho and Blitz, 1998; Ueki et al., 2003).

BMP7, a member of the TGF- $\beta$ superfamily, is widely expressed in thymus, bone marrow, spleen, brain, spinal cord, heart, skeletal muscle, kidney, lung, liver, pancreas, and prostate (Bragdon et al., 2011). BMP7 homozygous mutants exhibit many specific skeletal defects with variable penetrance, including abnormalities associated with development of the renal and ocular systems (Dudley et al., 1995). In the central nervous system (CNS), BMP7 regulates astrocytes. For example, Yanagisawa et al. (2001) have shown that BMP7 inhibits neurogenesis and induces astrocytogenesis of mouse fetal neuroepithelial cells. In the developing cerebral cortex, BMP7 induces radial glia differentiation and blocks neuronal migration (Ortega and Alcantara, 2010). Furthermore, Jordan et al. (1997) showed that application of BMP7 to rat mesencephalic cell cultures increased both the number of tyrosine hydroxylase-expressing cells and dopamine uptake. Moreover, BMP7 has been shown to promote dendritic growth in sympathetic neurons (Courter et al., 2016). These reports suggest that BMP7 plays pivotal roles in the CNS, but there is little information on the distribution of BMP7 in the adult CNS. Therefore, this distribution was investigated in this study.

\section{Experimental procedures}

\subsection{Animals and section preparation}

Wistar male rats ( $n=8,7$ weeks old; Japan SLC Inc., Shizuoka, Japan) of 7 weeks of age were used in the study. Under deep 
anesthesia, rats were perfused and fixed with $0.1 \mathrm{M}$ phosphate buffer ( $\mathrm{PB}, \mathrm{pH} 7.4$ ) containing $4 \%$ paraformaldehyde and $0.2 \%$ picric acid, and then the brains were quickly excised, postfixed with the same fixative solution for $2 \mathrm{~h}$ at $4{ }^{\circ} \mathrm{C}$, and immersed in $10 \%, 20 \%$, and $25 \%$ sucrose overnight at $4{ }^{\circ} \mathrm{C}$, respectively. Samples were frozen and cut using a cryostat. Sections for immunoperoxidase staining were $20 \mu \mathrm{m}$ thick and those for immunofluorescence staining were $10 \mu \mathrm{m}$. Serial sections of $5 \mu \mathrm{m}$ were used for the mirror method. This study was performed according to the Guidelines for Animal Experimentation at Hamamatsu University School of Medicine.

\subsection{Immunohistochemistry (IHC)}

For immunoperoxidase staining, slices were blocked with $10 \%$ normal goat serum, $2 \%$ bovine serum albumin (BSA) and $0.2 \%$ Triton X-100 in $0.1 \mathrm{M}$ PB for $2 \mathrm{~h}$ at room temperature (RT), and then incubated with rabbit anti-BMP7 antibody (diluted 1:500, final concentration, $2 \mu \mathrm{g} / \mathrm{ml}$; Sigma-Aldrich, St. Louis, MO) or rabbit anti-oxytocin antibody (diluted 1:30000, initial concentration not available; Immunostar, Hudson, WI) overnight at $4{ }^{\circ} \mathrm{C}$. After rinsing with $0.1 \mathrm{M} \mathrm{PB}$, the slices were incubated in goat anti-rabbit IgG with peroxidase complex (no dilution, ready-touse; EnVision $^{\mathrm{TM}}$ System, K4002; DAKO, Tokyo, Japan) for $2 \mathrm{~h}$ at RT. After rinsing with $0.1 \mathrm{M} \mathrm{PB}, 3,3$ '-diaminobenzidine (Wako, Osaka, Japan) was used as a substrate for visualization. IHC for controls was performed similarly, but without anti-BMP7 antibody.

For immunofluorescence staining with rabbit anti-BMP7 antibody (diluted 1:500; final concentration, $2 \mu \mathrm{g} / \mathrm{ml}$; Sigma-Aldrich), slices were blocked with $10 \%$ normal goat serum, $2 \%$ BSA and $0.2 \%$ Triton X-100 in $0.1 \mathrm{M}$ PB for $2 \mathrm{~h}$ at RT, and incubated with rabbit anti-BMP7 antibody overnight at $4{ }^{\circ} \mathrm{C}$. After rinsing with $0.1 \mathrm{M}$ $\mathrm{PB}$, slices were incubated with Alexa Fluor 594 goat anti-rabbit IgG (diluted 1:500; final concentration, $4 \mu \mathrm{g} / \mathrm{ml}$; Molecular Probes, Eugene, OR) for $1.5 \mathrm{~h}$ at RT. For double immunofluorescence staining with rabbit anti-BMP7 antibody and mouse anti-glial fibrillary acidic protein (GFAP) antibody (Millipore, Temecula, CA), slices were blocked with $10 \%$ normal goat serum, $2 \%$ BSA and $0.2 \%$ Triton X-100 in $0.1 \mathrm{M}$ PB for $2 \mathrm{~h}$ at RT, and then incubated with rabbit anti-BMP7 antibody (diluted 1:500; final concentration, $2 \mu \mathrm{g} / \mathrm{ml}$ ) and mouse anti-GFAP antibody (diluted 1:1000; initial concentration not available) overnight at $4{ }^{\circ} \mathrm{C}$. After rinsing with $0.1 \mathrm{M} \mathrm{PB}$, slices were incubated with both Alexa Fluor 594 goat anti-rabbit IgG (diluted 1:500; final concentration, $4 \mu \mathrm{g} / \mathrm{ml}$ ) and Alexa Fluor 488 goat anti-mouse IgG (diluted 1:500; final concentration, $4 \mu \mathrm{g} / \mathrm{ml}$; Molecular Probes) for $1.5 \mathrm{~h}$ at RT. For double immunofluorescence staining with rabbit anti-BMP7 antibody and goat anti-Iba1 antibody (Abcam plc, Cambridge, UK), slices were blocked with $10 \%$ normal donkey serum, $2 \%$ BSA and $0.2 \%$ Triton X-100 in $0.1 \mathrm{M}$ PB for $2 \mathrm{~h}$ at RT, and incubated with rabbit anti-BMP7 antibody (diluted 1:500; final concentration, $2 \mu \mathrm{g} / \mathrm{ml}$ ) and goat anti-Iba1 antibody (diluted 1:1000; final concentration, $1 \mu \mathrm{g} / \mathrm{ml}$ ) overnight at $4{ }^{\circ} \mathrm{C}$. After rinsing with $0.1 \mathrm{M} \mathrm{PB}$, slices were incubated with both Alexa Fluor 594 donkey anti-rabbit IgG (diluted 1:500; final concentration, $4 \mu \mathrm{g} / \mathrm{ml}$; Molecular Probes) and Alexa Fluor 488 donkey anti-goat IgG (diluted 1:500; final concentration, $4 \mu \mathrm{g} / \mathrm{ml}$; Molecular Probes) for $1.5 \mathrm{~h}$ at RT. At the end of the protocol, samples were mounted in ProLong Diamond Antifade Mountant with DAPI (Molecular Probes).

Bright field images were obtained using a microscope (Eclipse 80i; Nikon, Tokyo, Japan) equipped with a CCD camera (DS-Ri; Nikon). Fluorescence images were obtained using a microscope (Eclipse E-600; Nikon) equipped with the same CCD camera and further processed using Adobe Photoshop (Tokyo, Japan).

\subsection{Western blotting}

A whole rat brain was homogenized in $50 \mathrm{mM}$ Tris- $\mathrm{HCl}$ ( $\mathrm{pH}$ 7.4) buffer containing protease inhibitor cocktail (Nacalai Tesque, Kyoto, Japan) to avoid protein denaturation, and added to $2 \times$ SDS-PAGE sample buffer for solubilization. The sample was used for SDS-PAGE (14\% acrylamide; PAGEL, ATTO Corp., Tokyo, Japan). SDS-PAGE products were transferred to a PVDF membrane (Immobilon-P; Merck Millipore, Tokyo, Japan) by electroblotting ( $40 \mathrm{~V}$ overnight at $4{ }^{\circ} \mathrm{C}$ ) using a transfer buffer ( $25 \mathrm{mM}$ Tris, $192 \mathrm{mM}$ glycine) containing $20 \%$ methanol. The membranes were treated with $3 \%$ BSA in 50 mM Tris-buffered saline containing 0.05\% Tween20 (TTBS) for $2 \mathrm{~h}$ at RT and then incubated with rabbit anti-BMP7 antibody (diluted 1:600; final concentration, $1.67 \mu \mathrm{g} / \mathrm{ml}$; SigmaAldrich) in TTBS containing 1\% BSA for 40 min at RT. After rinsing with TTBS, the blots were incubated with horseradish peroxidaselinked goat anti-rabbit IgG (diluted 1:2000; final concentration, $1 \mu \mathrm{g} / \mathrm{ml}$; Nacalai Tesque) in TTBS containing $1 \%$ BSA for 40 min at RT. After rinsing the blots with TTBS several times, signals were visualized using a SuperSignal West Pico chemiluminescent Substrate (Thermo Fisher Scientific, Waltham, MA).

\section{Results}

Western blotting was performed to investigate whether the rabbit anti-BMP7 antibody specifically recognizes BMP7. As shown in Fig. $1 \mathrm{~A}$, western blotting with the antibody against whole rat brain gave a single band of about $48 \mathrm{kDa}$, which is consistent with the calculated size. IHC performed without the first antibody (Fig. 1B and C) showed no staining. These data clearly indicate that the rabbit anti-BMP7 antibody recognizes BMP7 protein.

\subsection{General expression patterns}

An overview of BMP7 expression in adult rat brain is shown in Fig. 2. BMP7-like immunoreactivity (IR) was seen throughout the brain, including abundant BMP7-IR in the olfactory bulb (Fig. 2A), basal ganglia (Fig. 2B), cerebral cortex (Fig. 2B-E), hippocampus (Fig. 2C-E), thalamus (Fig. 2C and D), hypothalamus (Fig. 2D and E), midbrain (Fig. 2E), cerebellum (Fig. 2F and G), brainstem (Fig. 2F-H), and spinal cord (Fig. 2I). A summary of the relative intensities of BMP7-IR in the rat CNS is given in Table 1.

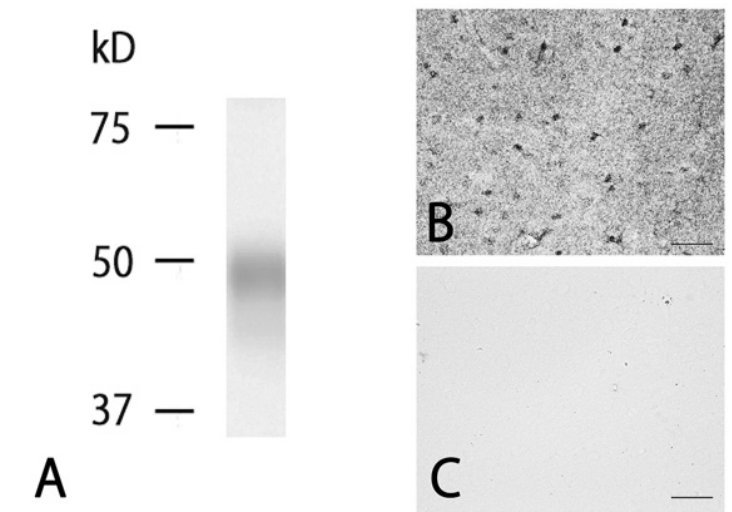

Fig. 1. Specificity of the anti-BMP7 antibody. Western blot analysis using anti-BMP7 antibody for whole rat brain gave a single band of about $48 \mathrm{kDa}(\mathrm{A})$. Immunostaining with anti-BMP7 antibody (B) without the first antibody (C) in the cerebral cortex. Immunostaining without the first antibody gave no significant signals. Scale bar $=40 \mu \mathrm{m}$ for $\mathrm{B}, \mathrm{C}$. 


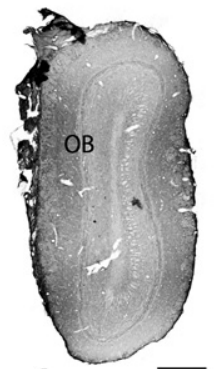

A
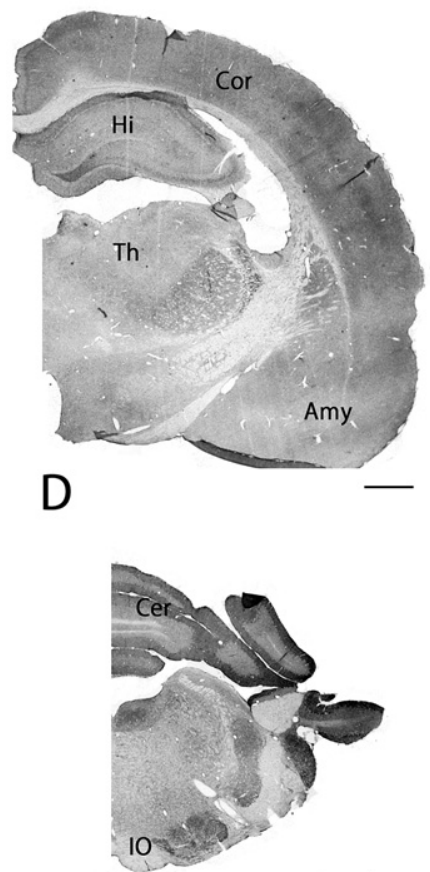

G
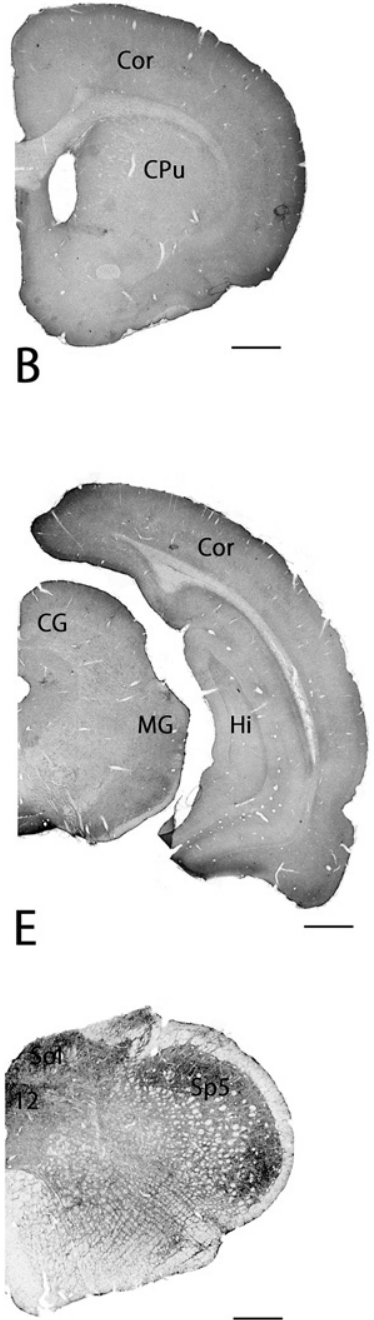

$\mathrm{H}$
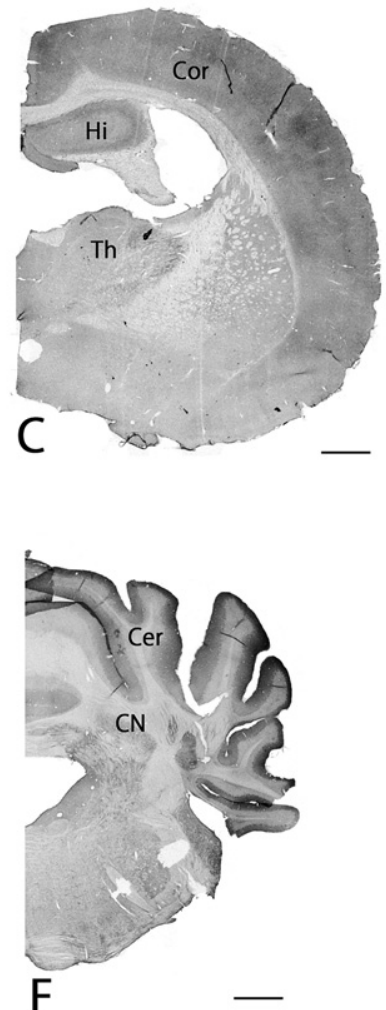

$\mathrm{F}$

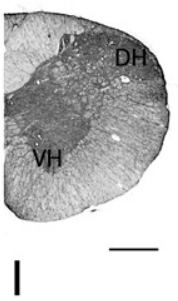

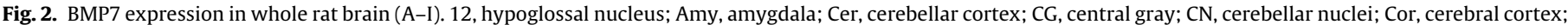

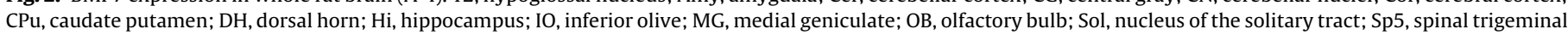
nucleus; Th, thalamus, VH, ventral horn. Scale bar $=0.5 \mathrm{~mm}$ for A, H, I; $1 \mathrm{~mm}$ for B-G.

\subsection{Telencephalon}

\subsubsection{Olfactory bulb}

Strong BMP7 expression was found in the olfactory nerve layer, with moderate expression in other layers (Fig. 3A). Closer observation showed strongly stained astrocyte-like cells (arrowheads in Fig. 3B) and moderately stained neuropils (Fig. 3B) in the external plexiform layer.

\subsubsection{Septum and nuclei of the diagonal band of Broca}

Moderate BMP7-IR was seen in the medial septal nuclei and diagonal band of Broca (Fig. 3C). Closer observation showed that small cells were moderately stained, and moderate neuropil staining was also seen (Fig. 3D and E). In the lateral septal nucleus, small BMP7-IR positive astrocyte-like cells were evenly distributed (arrowheads in Fig. 3F). Interestingly, strong BMP7-IR was seen on ependyma of lateral ventricles (arrows in Fig. 3F). This strong ependymal staining was also observed using immunofluorescent staining (arrows in Fig. 3G).

\subsubsection{Islands of Calleja}

Many moderately stained cells were detected in the islands of Calleja and moderately stained neuropils were seen (Fig. $3 \mathrm{H}$ ).

\subsubsection{Cerebral cortex}

Evenly distributed small cells strongly stained with BMP7 antibody and moderate neuropil staining were seen throughout the cerebral cortex (Fig. 4A). Closer observation showed that the small cells had an astrocyte-like appearance in layers I and V (arrowheads in Fig. 4B and C).

\subsubsection{Hippocampus}

Small BMP7-IR positive cells and weak to moderate neuropil staining were observed throughout the hippocampus (Fig. 4D). Closer observation showed strong BMP7-IR positive astrocyte-like cells in Ammon's horn (arrowheads in Fig. 4E and F); in contrast, BMP7-IR was not detected in pyramidal neurons (asterisks in Fig. 4F). Moderate neuropil staining was seen in the oriens layer (Fig. 4E and F). The dentate gyrus had strong BMP7-IR positive astrocyte-like cells (arrowheads in Fig. 4G and H), but BMP7-IR was 
Table 1

Distribution and Intensity of BMP7-IR in the rat CNS.

\begin{tabular}{|c|c|c|c|}
\hline Area & Intensity & Area & Intensity \\
\hline I. Telencephalon & & IV. Pons and medulla & \\
\hline Olafactory bulb & & Motor system & \\
\hline Olfactory nerve layer & +++ & Trigeminal motor nucleus & +++ \\
\hline Glomerular layer & ++ & Hypoglossal nucleus & +++ \\
\hline External plexiform layer & ++ & General somatosensory system & \\
\hline Mitral cell layer & ++ & Trigeminal mesencephalic nucleus & +++ \\
\hline Granular layer & ++ & Trigeminal spinal nucleus & +++ \\
\hline Subependymal layer & ++ & General visceromotor system & \\
\hline Cerebral cortex & ++ & Dorsal nucleus of the vagus & ++ \\
\hline Hippocampal formation & & General viscerosensory system & \\
\hline Ammon's horn & ++ & The nucleus of the solitary tract & ++ \\
\hline Dentate gyrus & +++ & Special somatosensory system & \\
\hline Amygdala & + & Auditory system & \\
\hline Basal ganglia & & Dorsal cochlear nucleus & ++ \\
\hline Caudate putamen & ++ & Ventral cochlear nucleus & +++ \\
\hline Globus pallidus & ++ & Inferior colliculus & ++ \\
\hline Ilands of Calleja & ++ & Vestibular system & \\
\hline Corpus callosum & +++ & Medial vestibular nucleus & +++ \\
\hline II. Diencephalon & & Pontine dorsal tegmental nucleus & +++ \\
\hline Thalamus & & Pontine nucleus & +++ \\
\hline Reticular nucleus & +++ & Area postrema & ++++ \\
\hline Medial habenular nucleus & ++ & Locus coeruleus & ++++ \\
\hline Lateral habenular nucleus & + & Inferior olive & +++ \\
\hline Ventroposterior nucleus & ++ & V. Cerebellum & \\
\hline Lateral geniculate nucleus & ++ & Cortex & \\
\hline Other nuclei & + & Molecular layer & ++++ \\
\hline Hypothalamus & & Purkinje cell layer & ++++ \\
\hline Paraventricular hypothalamic nucleus & +++ & Granule cell layer & ++ \\
\hline Other nuclei & + & Cerebellar nuclei & +++ \\
\hline Median eminence & ++++ & VI. Spinal cord & \\
\hline III. Midbrain & & Dorsal horm & +++ \\
\hline Red nucleus & ++ & Ventral horn & +++ \\
\hline Substantia nigra & ++ & & \\
\hline Interpeduncular nucleus & ++++ & & \\
\hline Oculomotor nucleus & +++ & & \\
\hline Superior colliculus & ++ & & \\
\hline
\end{tabular}

Relative intensities were estimated by visual comparison of immunostained slide: +, low; ++, moderate; +++, strong; ++++, very strong.

not seen in granule neurons (asterisks in Fig. 4H). Strong neuropil staining was seen in the polymorphic layer (Fig. 4G and H).

\subsubsection{Basal ganglia}

The caudate putamen had moderately positive small cells and moderate neuropil staining in gray matter (Fig. 5A and B), while the globus pallidus had moderately positive astrocyte-like cells (arrowheads in Fig. 5C) and weak neuropil staining in gray matter (Fig. 5C).

\subsubsection{Corpus callosum}

Strong BMP7-IR-positive cells were detected in the corpus callosum (arrowheads in Fig. 5D). On closer observation, these cells had an astrocyte-like appearance (arrowheads in Fig. 5E).

\subsubsection{Amygdala}

Weak BMP7-IR was seen in all nuclei of the amygdala (Fig. 2D).

\subsection{Diencephalon}

BMP7-IR positive cells and neuropils were observed in all nuclei in the thalamus (Fig. 2C and D, Fig. 5F). There were strongly positive astrocyte-like cells (arrowheads in Fig. 5G) and moderate neuropil staining in the reticular thalamic nucleus (Fig. 5G); strongly positive astrocyte-like cells and moderate neuropil staining in the ventroposterior thalamic nucleus (Fig. 5H); moderate BMP7-IR positive astrocyte-like cells and neuropils in the medial habenular nucleus (Fig. 5I and J); and moderately-stained small cells and neuropils in the lateral geniculate nucleus (Fig. $5 \mathrm{~K}$ and L).
In the hypothalamus, we detected weak BMP7-IR in all nuclei (Fig. 2D and E). Interestingly, the magnocellular part of the paraventricular hypothalamic nucleus contained very strongly positive neurons (arrows in Fig. 6A). Closer observation showed that large neurons with varicose neurites exhibited very strong BMP7-IR (Fig. 6A and B). To determine which kinds of neurons express BMP7-IR, we performed the mirror method, in which pairs of consecutive thin sections were stained with BMP7 antibody and rabbit anti-oxytocin antibody, respectively, and found that the positive neurons are oxytocin neurons (arrowheads in Fig. $6 C_{1}, C_{2}$ ). In addition, the medial part of the median eminence showed very strong BMP7-IR (arrows in Fig. 6D). Closer observation showed many strongly stained astrocyte-like cells expressed abundant BMP7 proteins (arrowheads in Fig. 6E). This strong BMP7-IR in the median eminence was also observed using immunofluorescent staining (arrowheads in Fig. 6F).

\subsection{Midbrain}

Very strong BMP7-IR was detected in the interpeduncular nucleus (arrows in Fig. 7A). On closer observation, very strongly stained astrocyte-like cells (arrowheads in Fig. 7B) and very strong neuropil staining were detected (Fig. 7B). In addition, the red nucleus and substantia nigra were moderately stained (Fig. 7A). In the red nucleus, large-sized neurons exhibited strong BMP7-IR (arrowheads in Fig. 7C), while weak BMP7-IR was detected in neuropils (Fig. 7C). Strongly-stained neurons (arrowheads in Fig. 7D) and weak neuropil staining (Fig. 7D) were observed in the pars compacta of the substantia nigra. The superior colliculus showed 

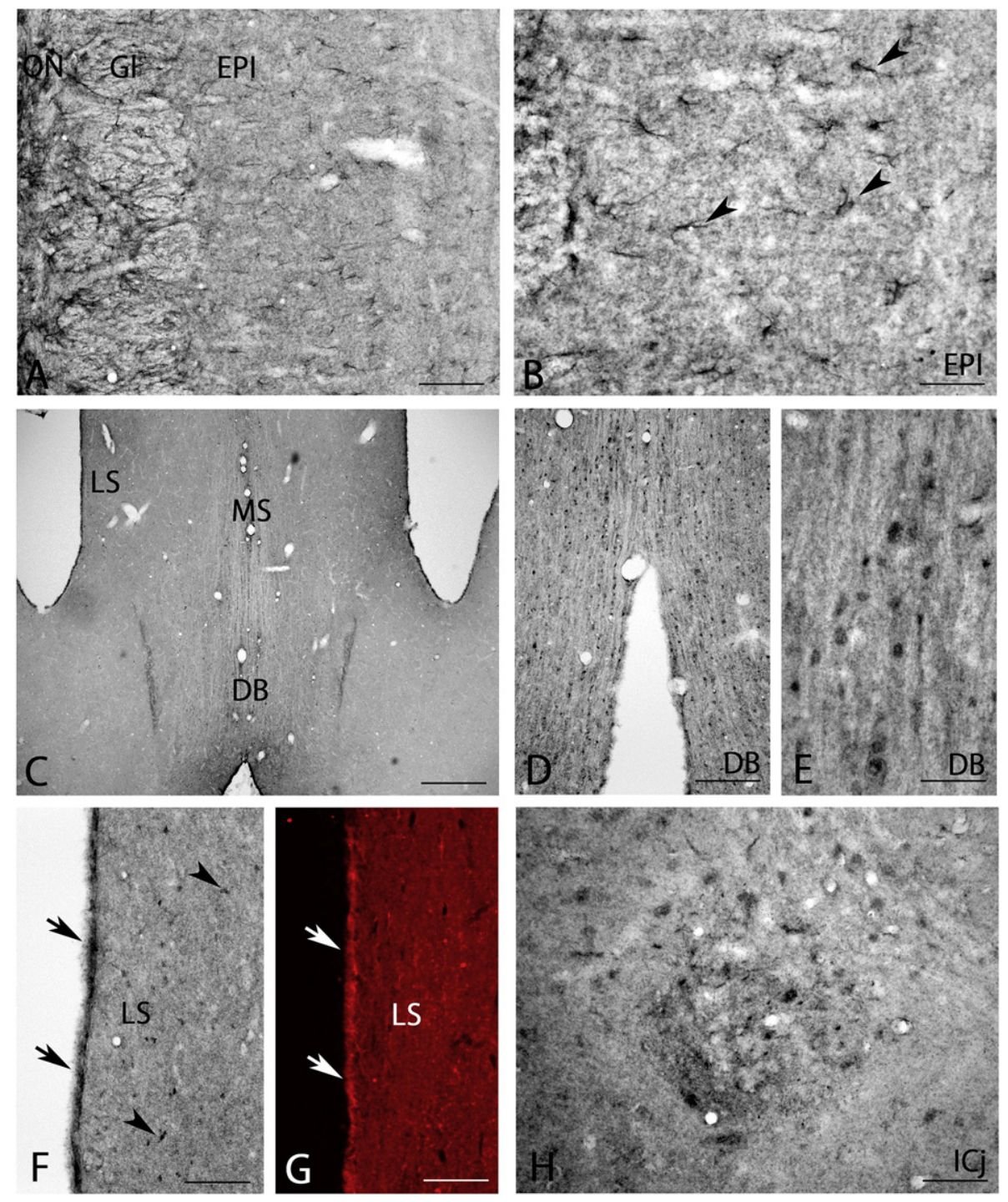

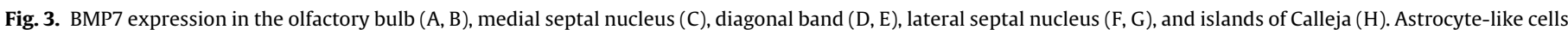

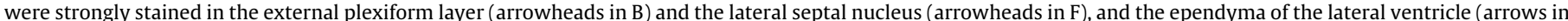

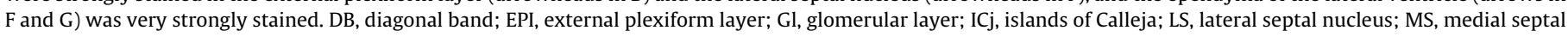
nucleus; ON, olfactory nerve layer. Scale bar $=160 \mu \mathrm{m}$ for C; $80 \mu \mathrm{m}$ for A, D, F; $40 \mu \mathrm{m}$ for B, E, G, H.

moderate BMP7-IR (Fig. 7E). Interestingly, the superficial part of the superior colliculus showed strong BMP7-IR (Fig. 7E). In addition, strong BMP7-IR was observed in the pia mater of the superior colliculus (arrows in Fig. 7E). This strong BMP7-IR in the pia mater was also observed using immunofluorescent staining (arrows in Fig. 7F). Furthermore, the oculomotor nucleus exhibited abundant BMP7-IR in neuronal cell bodies and neuropils (Fig. 7G).

\subsection{Pons and medulla}

\subsubsection{Motor system}

Nuclei in the general somatomotor and brachiomotor system, such as the motor trigeminal nucleus (Fig. $7 \mathrm{H}$ ) and hypoglossal nucleus (Fig. 7J), showed abundant BMP7-IR in neuronal cell bodies and neuropils. Interestingly, the facial nerve showed strong BMP7IR in axon fibers (arrows in Fig. 7I).

\subsubsection{General somatosensory system}

Large primary afferent neurons were strongly stained in the trigeminal mesencephalic nucleus (arrowheads in Fig. 7K), with scattered strongly stained astrocyte-like cells (Fig. 7K). The trigeminal spinal nucleus had strongly stained small cells and neuropils (Fig. 7L, M), and strongly stained axons were also seen in the medial lemniscus (arrows in Fig. 7N).

\subsubsection{General visceromotor system}

Moderate BMP7-IR positive neurons and weak neuropil staining were observed in the dorsal nucleus of the vagus (Fig. 8A).

\subsubsection{General viscerosensory system}

Moderate BMP7-IR small cells and moderate neuropil staining were seen in the nucleus of the solitary tract (Fig. 8A and B).

\subsubsection{Special somatosensory system}

Auditory system: Ventral cochlear nuclei had strong BMP7-IR staining (Fig. 8C) and dorsal cochlear nuclei had moderate BMP7-IR (Fig. 8D). Moderate BMP7-IR was detected in the inferior colliculus and strong BMP7-IR staining was seen in the superior olive (Fig. 8E).

Vestibular system: Strongly stained axons were seen in the vestibulocochlear nerve (arrows in Fig. 8F) and there was strong 

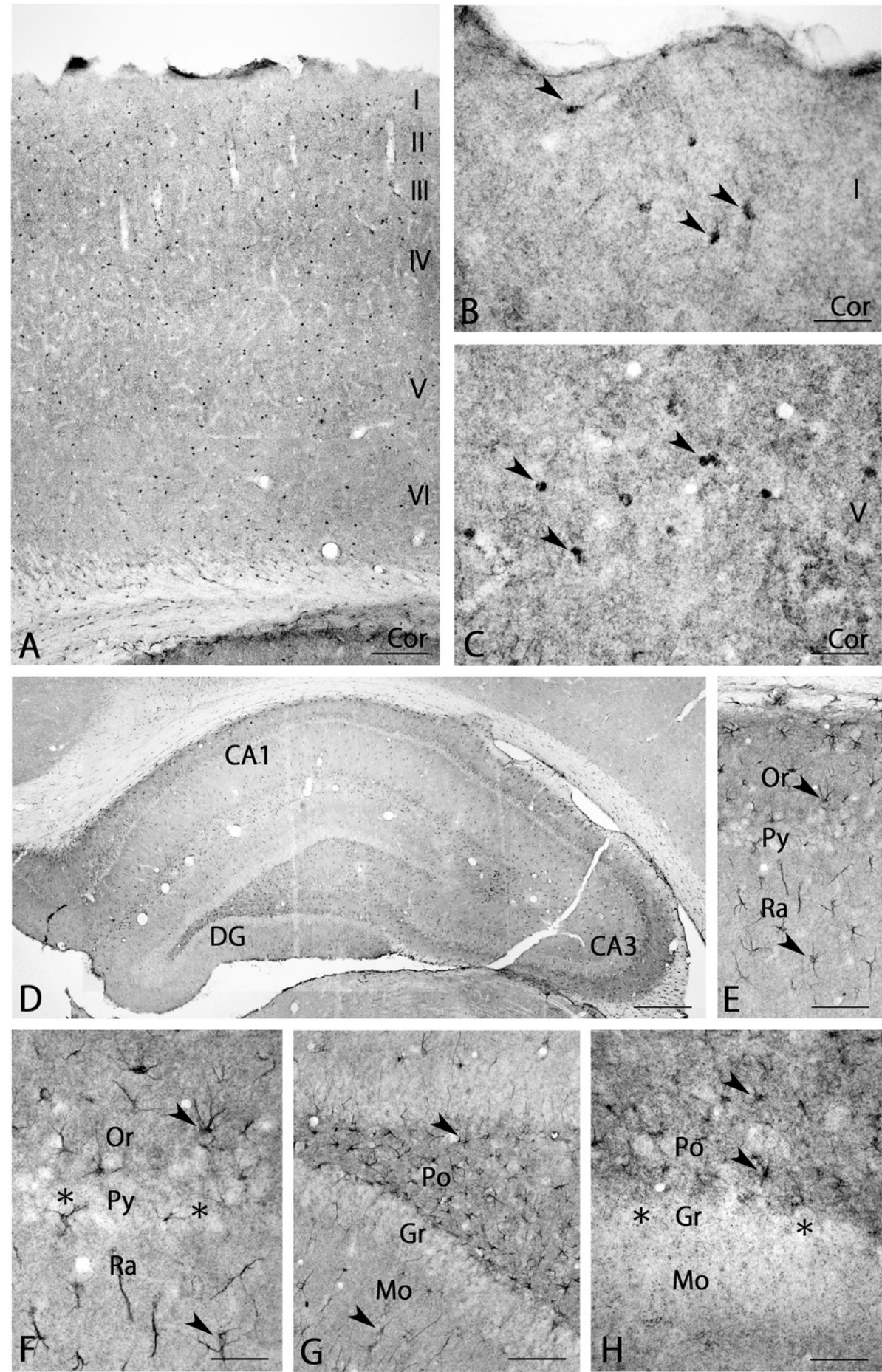

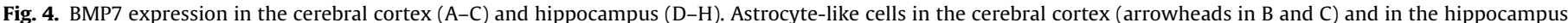

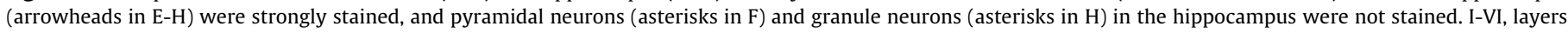

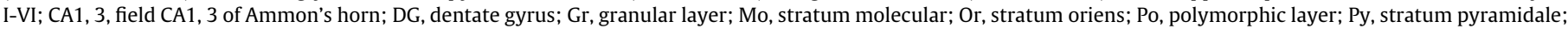
$\mathrm{Ra}$, stratum radiatum. Scale bar $=400 \mu \mathrm{m}$ for D; $160 \mu \mathrm{m}$ for A; $80 \mu \mathrm{m}$ for E, G; $40 \mu \mathrm{m}$ for B, C, F, H.

BMP7-IR in the medial vestibular nucleus (Fig. 8G) and in the ependyma of the fourth ventricle (arrowheads in Fig. 8G). This strong ependymal BMP7-IR was also seen using immunofluorescent staining (arrows in Fig. 8H).

\subsubsection{Other lower brain stem areas}

Strong BMP7-IR was observed in the pontine dorsal tegmental nucleus (Fig. 9A) and pontine nucleus (Fig. 9B). Closer observation also showed strongly-stained small cells and neuropils in the pontine nucleus (Fig. 9B and C). In addition, very strong BMP7-IR was observed in the area postrema (Fig. 9D) and there were very strongly stained neurons and neuropils in the locus coeruleus (Fig. 9E), and strongly stained neurons and moderate neuropil staining in the inferior olive (Fig. 9F and G). Interestingly, there were also many BMP7-IR axons in the inferior cerebellar peduncle, which originates from the inferior olive (arrows in Fig. 9H). 

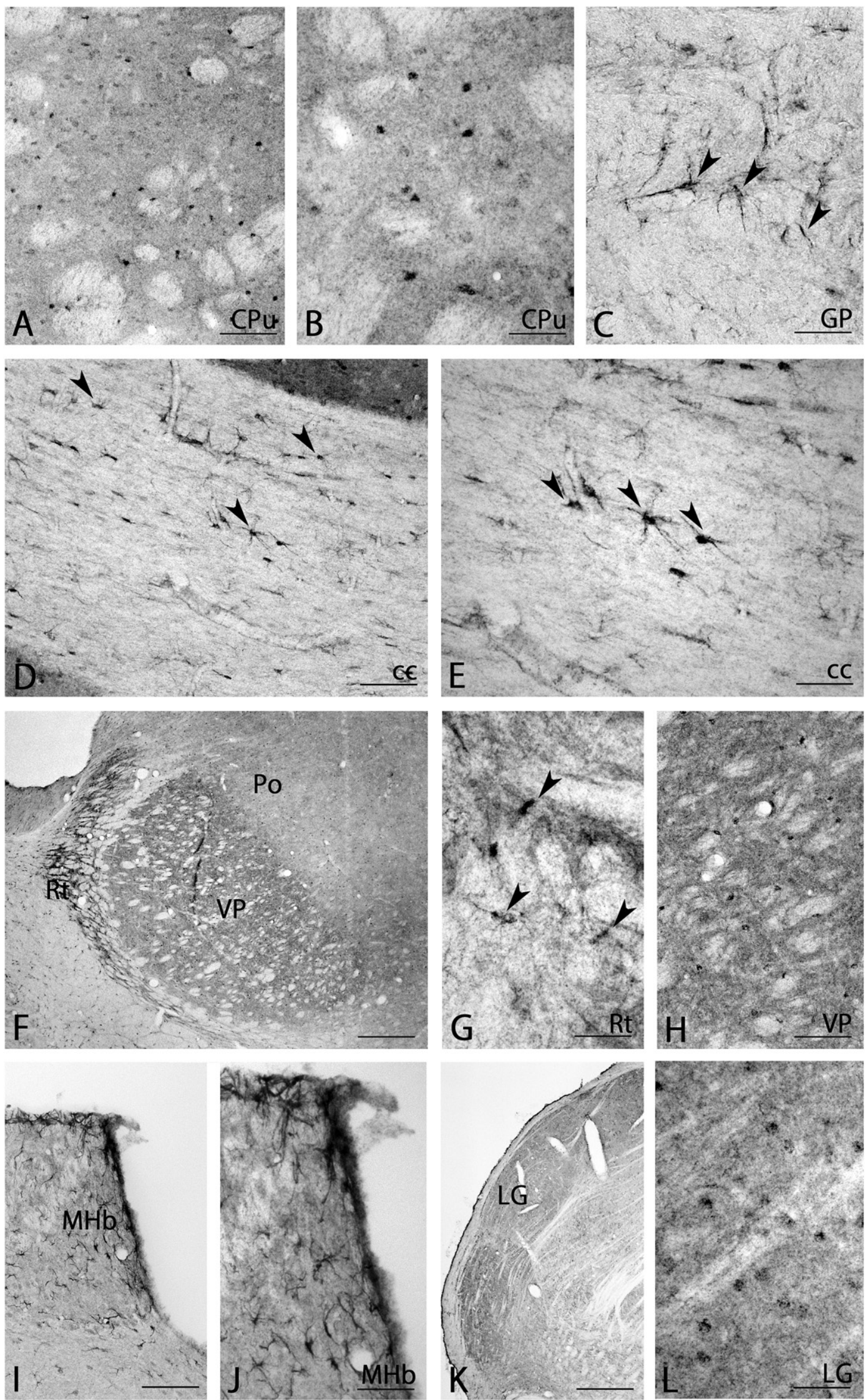

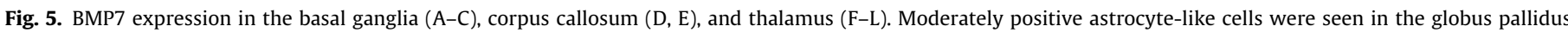

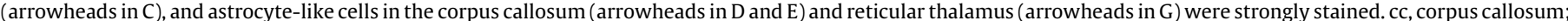

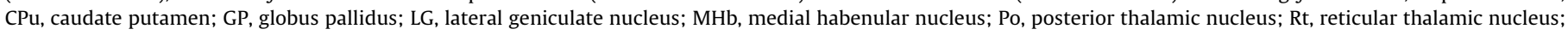
$\mathrm{VP}$, ventroposterior thalamic nucleus. Scale bar $=400 \mu \mathrm{m}$ for F, K; $80 \mu \mathrm{m}$ for A, D, H, I; $40 \mu \mathrm{m}$ for B, C, E, G, J, L. 

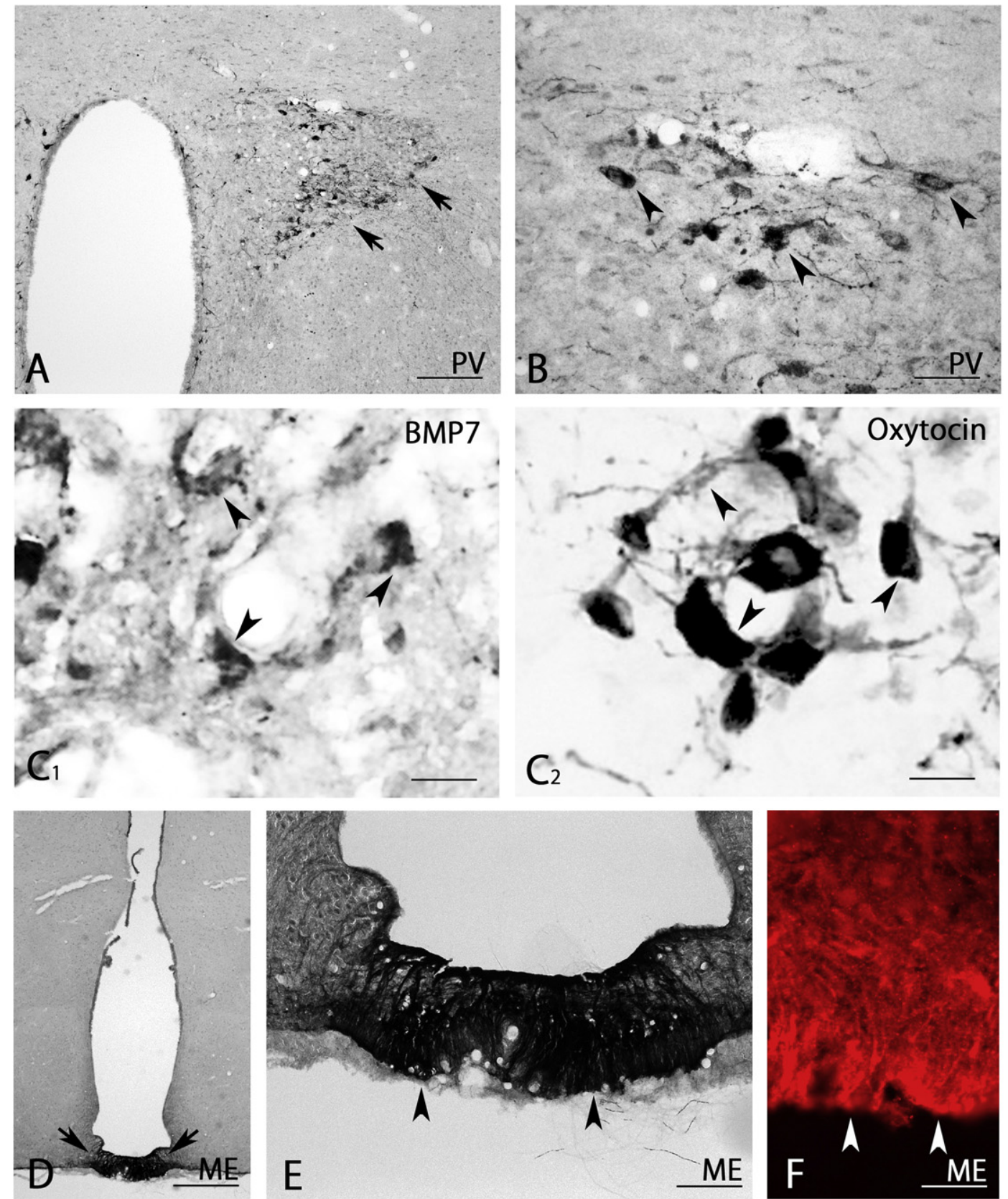

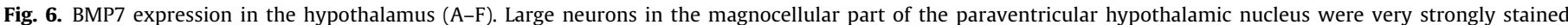

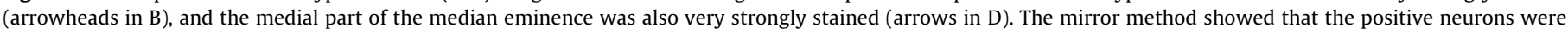

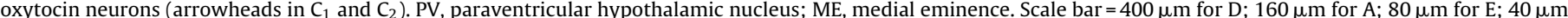
for $\mathrm{B} ; 20 \mu \mathrm{m}$ for $\mathrm{C}_{1}, \mathrm{C}_{2}, \mathrm{~F}$.

\subsection{Cerebellum}

There was very strong BMP7-IR in the Purkinje cell layer and the molecular cell layer in the cerebellum. Cell bodies of Purkinje neurons (arrows in Fig. 10A) and Bergmann glias around these cell bodies exhibited very strong BMP7-IR (arrowheads in Fig. 10A). Moderate BMP7-IR was seen in the granular cell layer (Fig. 10A). Cerebellar nuclei had strong BMP7-IR positive neurons (arrowheads in Fig. 10B) and moderate neuropil staining (Fig. 10B).

\subsection{Spinal cord}

There was strong BMP7-IR in the gray matter of the spinal cord, while BMP7 expression in the white matter was relatively weak
(Fig. 10C). Strong positive cells and neuropils were detected in the dorsal horn (Fig. 10D) and the ventral horn (Fig. 10E). Closer observation showed that large motor neurons expressed BMP7IR (arrowheads in Fig. 10F). Astrocyte-like cells (arrowheads in Fig. 10G) and axons (arrows in Fig. 10G) were stained in the white matter.

\subsection{Other areas}

In the subventricular zone (SVZ), moderately stained astrocytelike cells were detected (arrowheads in Fig. 10H). Ependymal cells were moderately stained (arrows in Fig. 10H) and the choroidal plexus also expressed abundant BMP7 (arrowheads in Fig. 10I). 

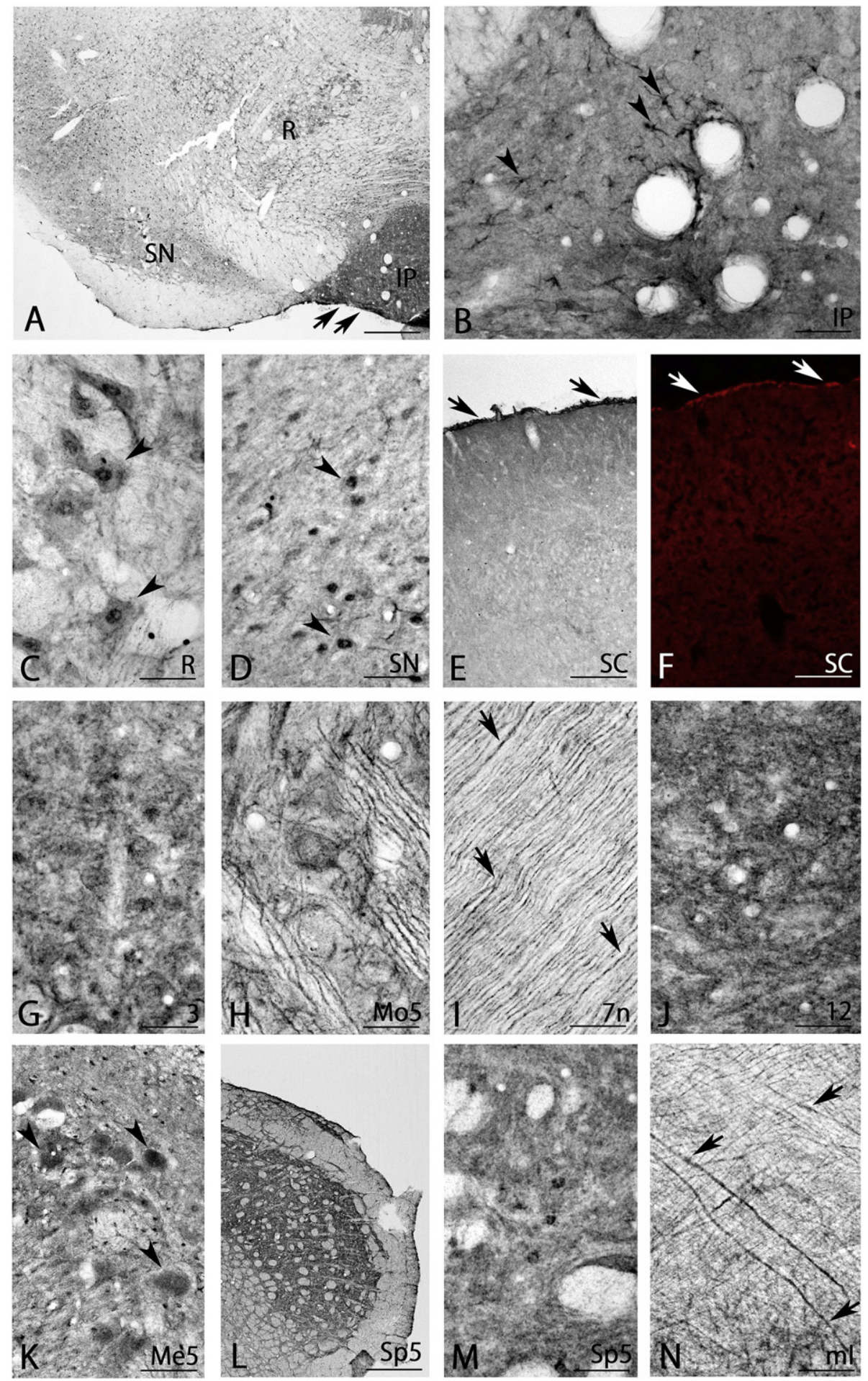

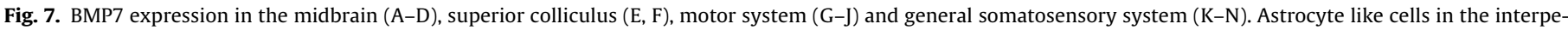

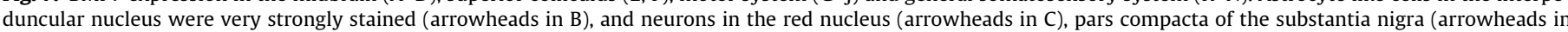

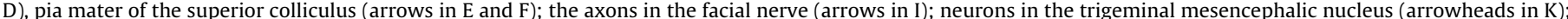

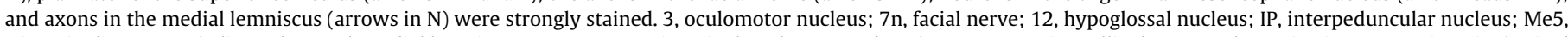

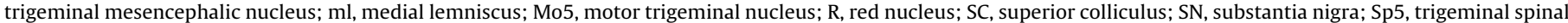
nucleus. Scale bar $=400 \mu \mathrm{m}$ for A; $160 \mu \mathrm{m}$ for E, L; $80 \mu \mathrm{m}$ for F; $40 \mu \mathrm{m}$ for B-D, G-K, M, N.

\subsection{Double fluorescence immunohistochemistry}

Many astrocyte-like cells were detected throughout the CNS. To show that these cells are actually astrocytes, double fluorescence IHC with anti-GFAP antibody was performed in the cerebral cortex, hippocampus, corpus callosum and cerebellum. As shown in Fig. 11A-D, co-expression of BMP7 and GFAP was observed in these regions, indicating that astrocytes express BMP7. To further examine BMP7 expression in astrocytes in the cortex, hippocampus, and corpus callosum, we combined nuclear staining using 

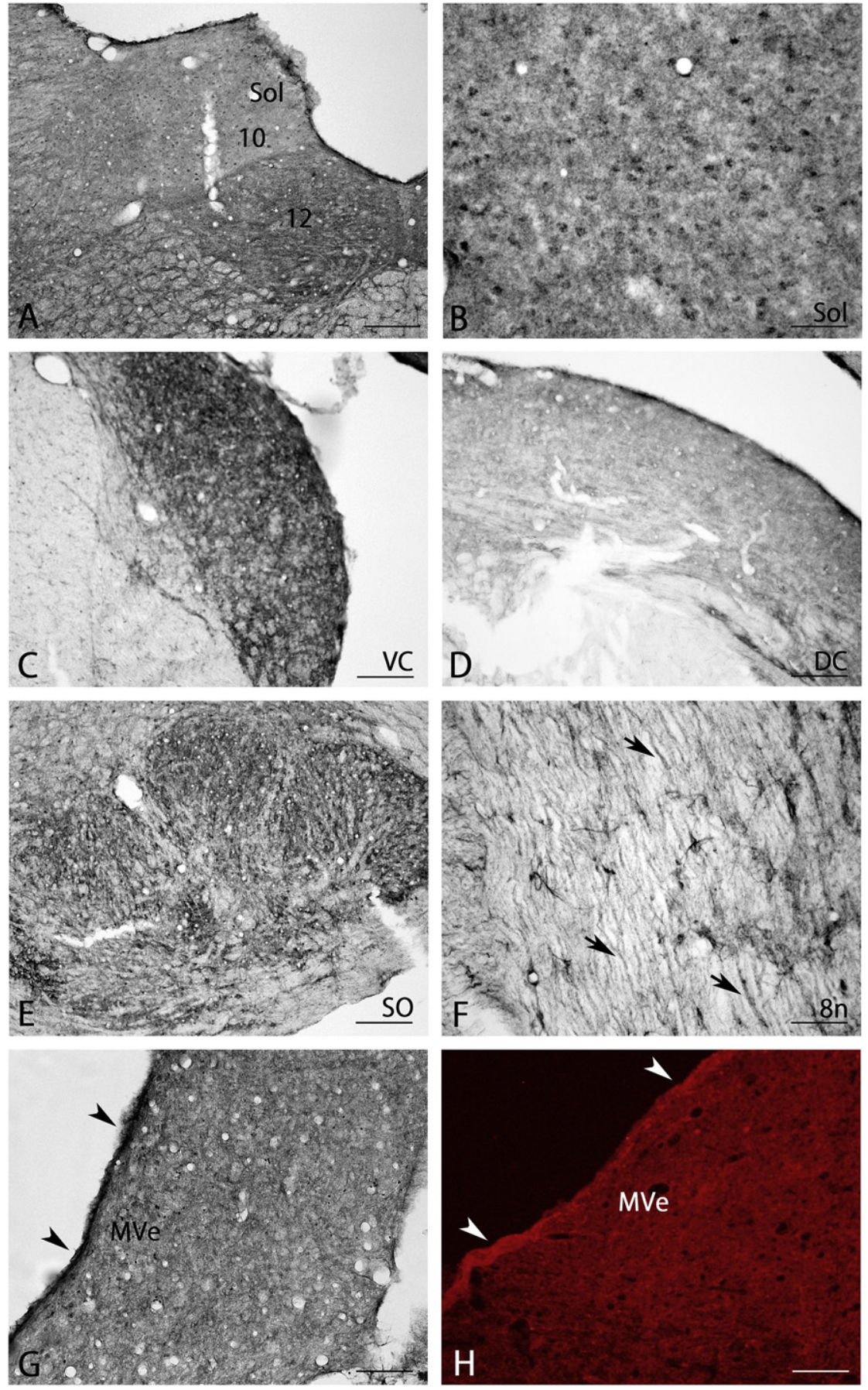

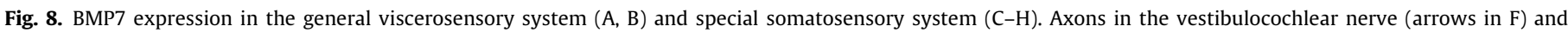

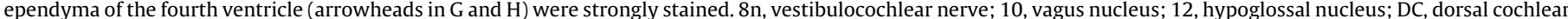

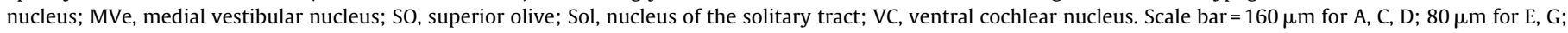
$40 \mu \mathrm{m}$ for B, F, H.

4', 6-diamidino-2-phenylindole (DAPI) with double fluorescence IHC with anti-GFAP antibody. In these regions, BMP7-IR was only detected in cells positive for GFAP (Fig. 12A-C), further indicating that astrocytes express BMP7. Double fluorescence IHC with anti-Iba1 antibody (microglial marker) was also performed, but Iba1-positive microglial cells did not show any BMP7-IR (Fig. 12D).

\section{Discussion}

In this study, we showed that BMP7 is widely expressed in the adult CNS. Interestingly, abundant BMP7 expression in astrocytes was found throughout the CNS, in addition to staining for BMP7 in certain neuronal cell bodies, axons, ependymal cells and meninges.

\subsection{BMP7 expression in astrocytes}

Strong BMP7 expression in astrocytes was observed throughout the CNS. In the cortex and hippocampus, many strongly stained astrocytes were present, but BMP7 expression in neurons was not detected. These results suggest that astrocytes are the main suppliers of BMP7 in the CNS. Interestingly, BMP7 is known to regulate astrocytes. For example, Yanagisawa et al. (2001) found that BMP7 

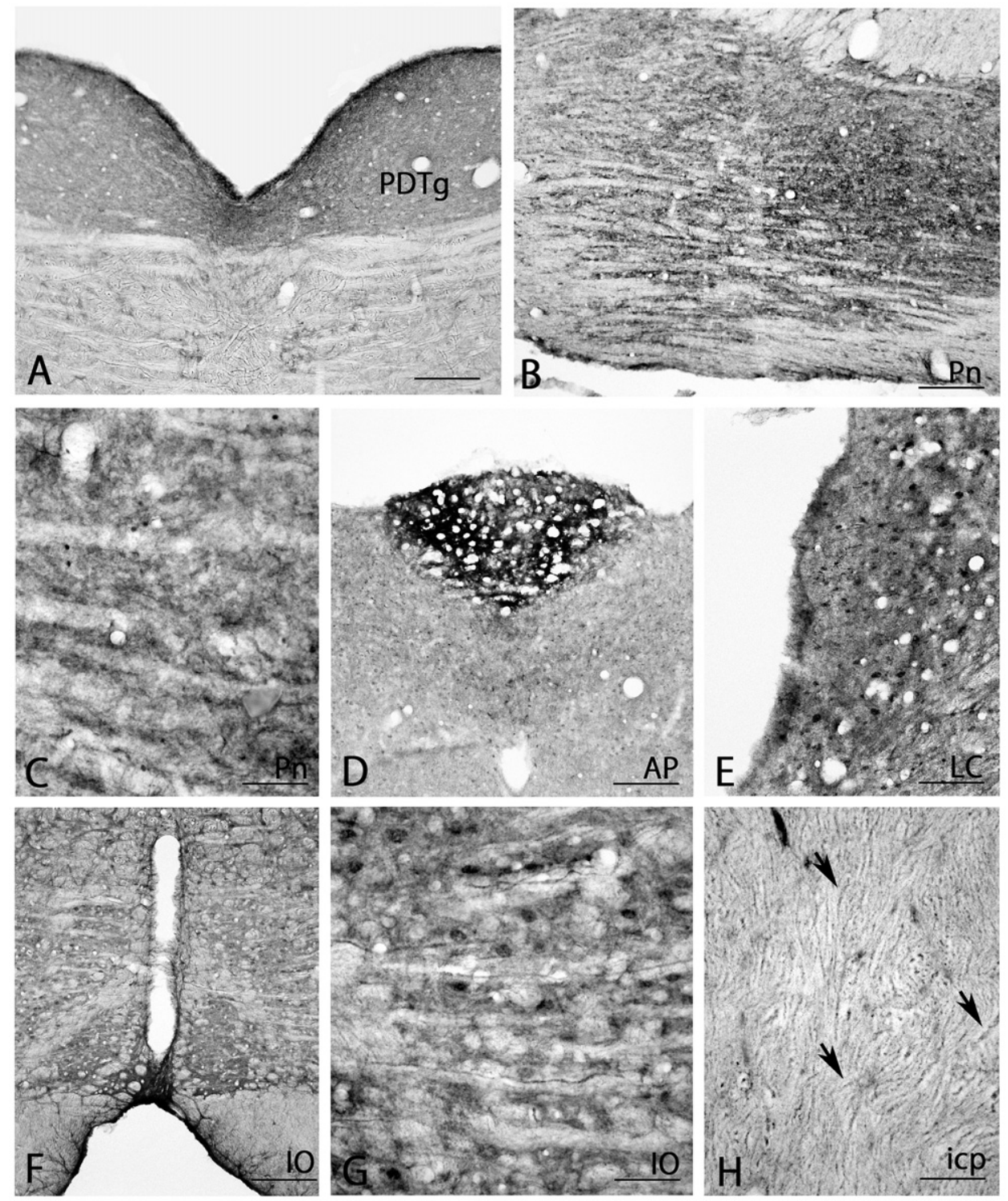

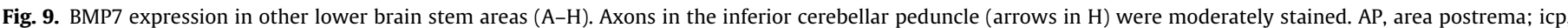

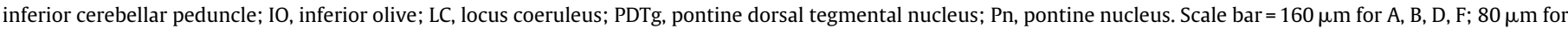
E; $40 \mu \mathrm{m}$ for C, G, H.

inhibits neurogenesis and concomitantly induces astrocytogenesis of mouse fetal neuroepithelial cells. In addition, in the developing cerebral cortex, BMP7 induces premature radial glia differentiation and impairs neuronal migration (Ortega and Alcantara, 2010). These reports show that BMP7 is highly involved in differentiation of astrocytes.

BMP7 is also involved in gliosis. For example, BMP7 increases the levels of proteins associated with reactive gliosis in retinal astrocytes and Müller glial cells, including GFAP, glutamine synthetase, a subset of chondroitin sulfate proteoglycans (CSPGs), matrix metalloproteinases, and other molecules (Dharmarajan et al., 2014). Local increases in BMP7 at the site of a demyelinating lesion cause upregulation of gliosis, glial scar formation, and increased levels of CSPGs such as neurocan and aggrecan, which may inhibit remyelination (Fuller et al., 2007). Taken together, these results suggest that BMP7 released from astrocytes may then regulate the astrocytes themselves under normal and pathological conditions.

\subsection{BMP7 expression in neurons}

BMP7 was not detected in many kinds of neurons, including those in the cerebral cortex and hippocampus, but was found abundantly in oxytocin, noradrenergic and dopamine neurons. Liu and Placzek (2014) found that BMP7 acts as a cue to direct hypothalamic neurosecretory axons into the medial ventral midline of the hypothalamus through repulsion. Interestingly, we found intense BMP7 expression in oxytocin neurons in the magnocellular part of the paraventricular hypothalamic nucleus and astrocytes in the medial part of the median eminence (Fig. 6). Since axons of oxytocin and vasopressin neurons pass through this medial region, BMP7 in oxytocin neurons and the medial part of the medial eminence may be necessary to keep the trajectories of axons of these neurons to the posterior lobe of the pituitary.

BMP7 is also a critical mediator of catecholaminergic neuron differentiation. Deletion of BMP7 and BMP5 genes results in an absence of noradrenergic neurons in the locus coeruleus 

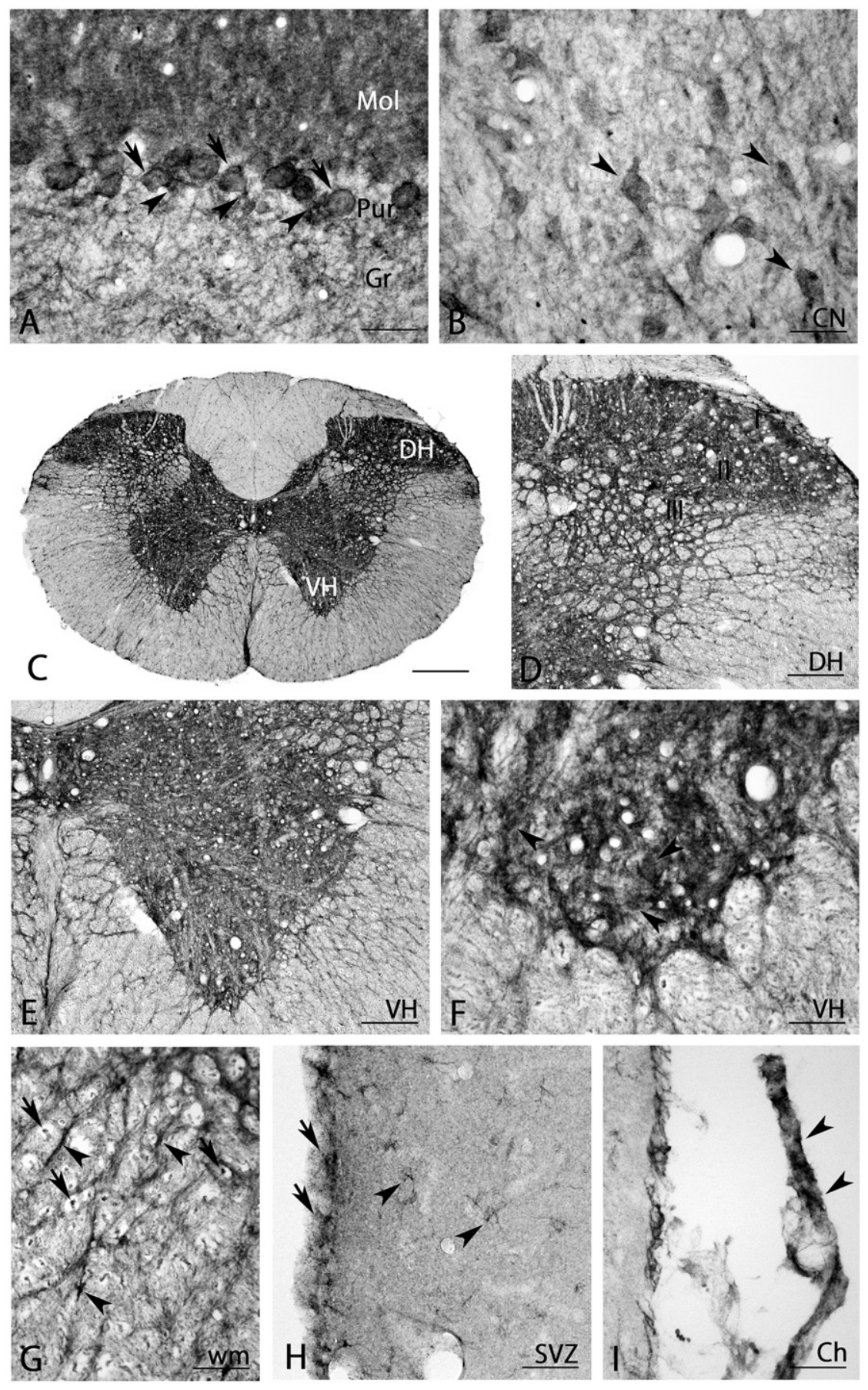

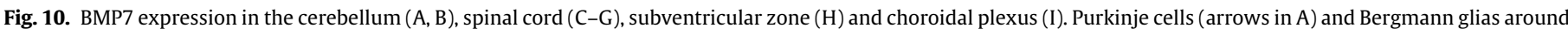

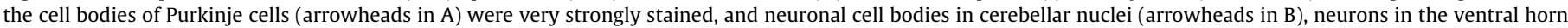

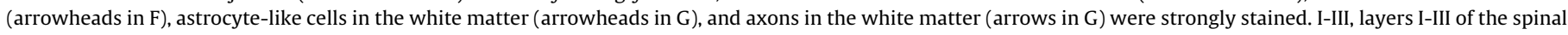

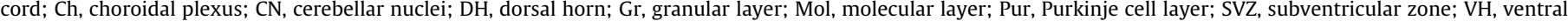
horn; wm, white matter. Scale bar $=400 \mu \mathrm{m}$ for C; $80 \mu \mathrm{m}$ for D, E; $40 \mu \mathrm{m}$ for A, B, F, G-I.

(Tilleman et al., 2010). In addition, BMP7 induces dendritic growth of noradrenergic neurons (Lein et al., 1995, 2002) and protects these neurons against 6-hydroxydopamine-induced neurotoxicity (Harvey et al., 2004). Jordan et al. (1997) showed that application of BMP7 to rat mesencephalic cell cultures increases both the number of tyrosine hydroxylase-expressing cells and dopamine uptake. These reports show that BMP7 has important roles in noradrenergic and dopamine neurons, and may be needed to maintain the identities of these catecholaminergic neurons.

\subsection{BMP7 expression in axons}

BMP7 was expressed in many kinds of axons, such as these in the facial nerve (Fig. 7I) and vestibulocochlear nerve (Fig. 8F). Retrograde BMP signal transduction through axons has been shown to modulate neuronal terminal differentiation and synaptic efficacy (da Silva and Wang, 2011). In Drosophila, McCabe et al. (2003) found that a muscle-derived BMP homolog, Glass bottom boat (Gbb), participates in coordinating neuromuscular 

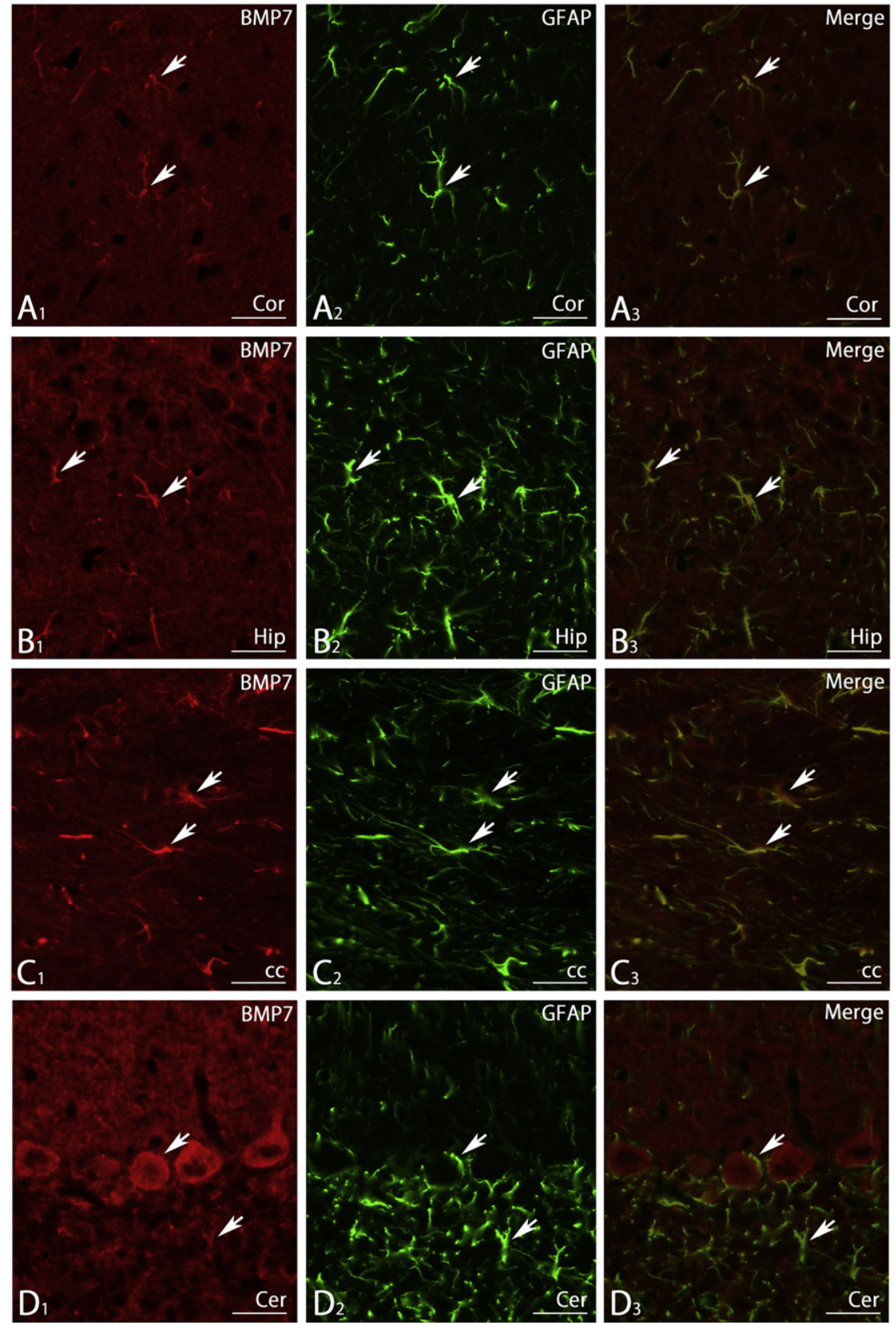

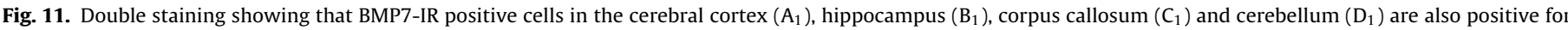
$\operatorname{GFAP}\left(A_{2}-D_{2}\right)$ in a merged photomicrograph $\left(A_{3}-D_{3}\right)$. cc, corpus callosum; Cer, cerebellum; Cor, cerebral cortex; Hip, hippocampus. Scale bar $=20 \mu m$.

synapse development and growth. In mice, target-derived BMP signaling controls development of trigeminal ganglia and sensory innervation of the skin (Guha et al., 2004), and regulates spatial patterns of gene expression in trigeminal ganglia (Hodge et al., 2007). These data suggest that BMP7 released from postsynaptic neurons or surrounding astrocytes may be taken up by presynapses and may function in retrograde signaling. In support of this speculative role for BMP7, Tsai et al. (2010) found that BMP7 overexpression mediated through adenoviral gene transfer to sciatic nerves protects against axonal and myelin degeneration and improves functional recovery after sciatic nerve injury in rats. 

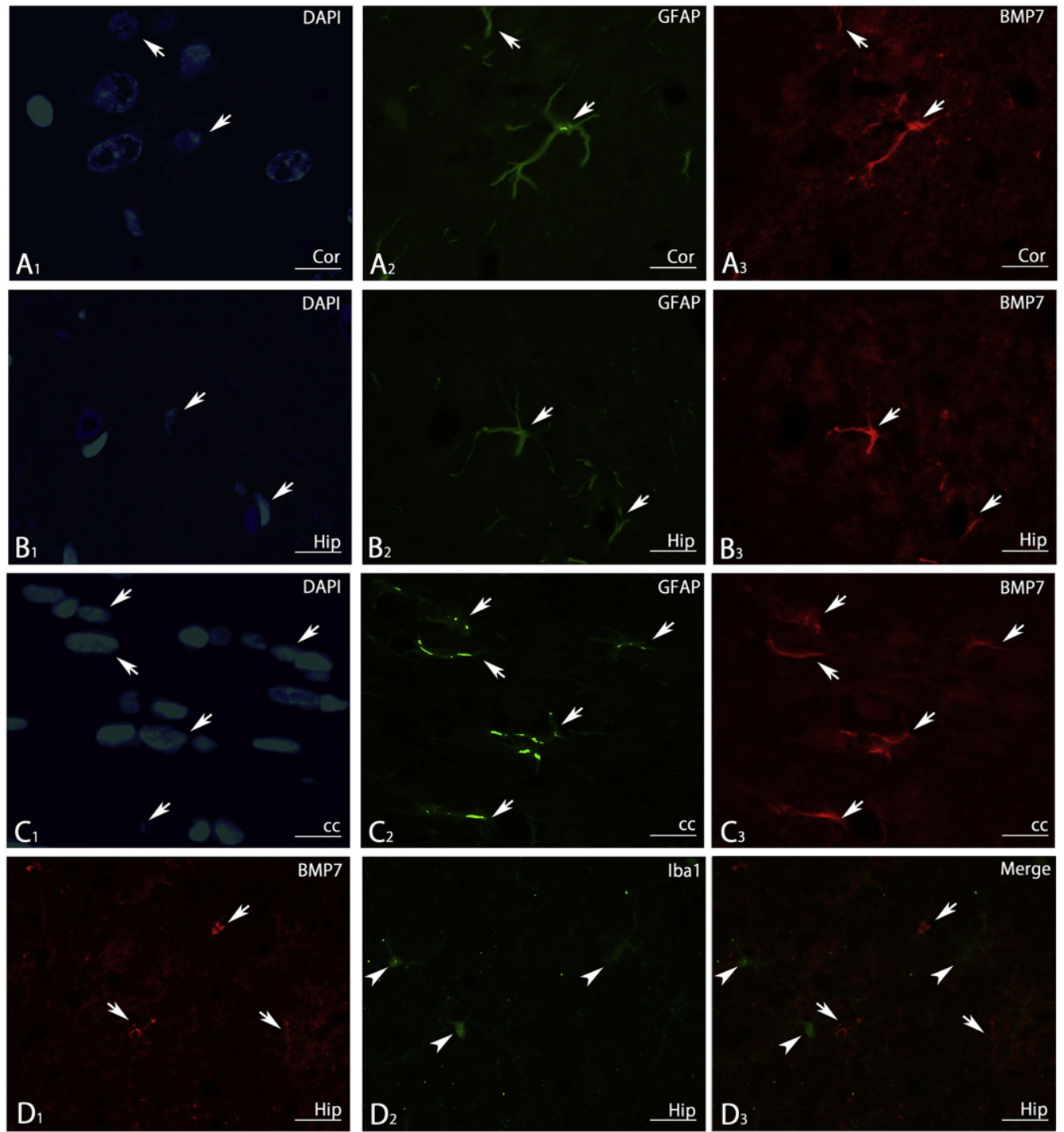

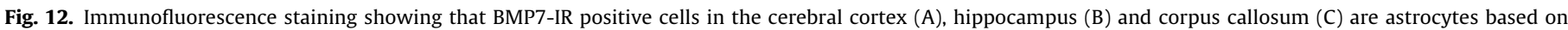

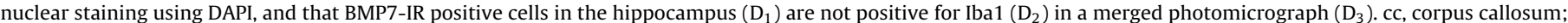
Cor, cerebral cortex; DAPI, 4', 6-diamidino-2-phenylindole; Hip, hippocampus. Scale bar = $20 \mu \mathrm{m}$ for D; $8 \mu \mathrm{m}$ for A-C.

\section{Acknowledgement}

The authors would like to thank Ms. Cong Wang (Henan University of Chinese Medicine) for her excellent technical help.

Grant sponsor: Grant-in-Aid for Scientific Research B, No. 15H04273 (Ministry of Education, Science and Culture of Japan).

\section{References}

Bragdon, B., Moseychuk, O., Saldanha, S., King, D., Julian, J., Nohe, A., 2011. Bone morphogenetic proteins: a critical review. Cell Signal. 23, 609-620.

Cho, K.W., Blitz, I.L., 1998. BMPs, Smads and metalloproteases: extracellular and intracellular modes of negative regulation. Curr. Opin. Genet. Dev. 8, 443-449.

Courter, L.A., Shaffo, F.C., Ghogha, A., Parrish, D.J., Lorentz, C.U., Habecker, B.A., Lein, P.J., 2016. BMP7-induced dendritic growth in sympathetic neurons requires p75(NTR) signaling. Dev. Neurobiol. 76, 1003-1013.

da Silva, S., Wang, F., 2011. Retrograde neural circuit specification by target-derived neurotrophins and growth factors. Curr. Opin. Neurobiol. 21, 61-67.

Dharmarajan, S., Gurel, Z., Wang, S., Sorenson, C.M., Sheibani, N., Belecky-Adams, T.L., 2014. Bone morphogenetic protein 7 regulates reactive gliosis in retinal astrocytes and Müller glia. Mol. Vis. 20, 1085-1108.

Dudley, A.T., Lyons, K.M., Robertson, E.J., 1995. A requirement for bone morphogenetic protein-7 during development of the mammalian kidney and eye. Genes Dev. 9, 2795-2807.
Fuller, M.L., DeChant, A.K., Rothstein, B., Caprariello, A., Wang, R., Hall, A.K., Miller, R.H., 2007. Bone morphogenetic proteins promote gliosis in demyelinating spinal cord lesions. Ann. Neurol. 62, 288-300.

Guha, U., Gomes, W.A., Samanta, J., Gupta, M., Rice, F.L., Kessler, J.A., 2004. Target-derived BMP signaling limits sensory neuron number and the extent of peripheral innervation in vivo. Development 131, 1175-1186.

Harvey, B.K., Mark, A., Chou, J., Chen, G.J., Hoffer, B.J., Wang, Y., 2004. Neurotrophic effects of bone morphogenetic protein-7 in a rat model of Parkinson's disease. Brain Res. 1022, 88-95.

Hodge, L.K., Klassen, M.P., Han, B.X., Yiu, G., Hurrell, J., Howell, A., Rousseau, G., Lemaigre, F., Tessier-Lavigne, M., Wang, F., 2007. Retrograde BMP signaling regulates trigeminal sensory neuron identities and the formation of precise face maps. Neuron 55, 572-586.

Jordan, J., Böttner, M., Schluesener, H.J., Unsicker, K., Krieglstein, K., 1997. Bone morphogenetic proteins: neurotrophic roles for midbrain dopaminergic neurons and implications of astroglial cells. Eur. J. Neurosci. 9, 1699-1710.

Lein, P., Johnson, M., Guo, X., Rueger, D., Higgins, D., 1995. Osteogenic protein-1 induces dendritic growth in rat sympathetic neurons. Neuron 15, 597-605.

Lein, P.J., Beck, H.N., Chandrasekaran, V., Gallagher, P.J., Chen, H.L., Lin, Y., Guo, X., Kaplan, P.L., Tiedge, H., Higgins, D., 2002. Glia induce dendritic growth in cultured sympathetic neurons by modulating the balance between bone morphogenetic proteins (BMPs) and BMP antagonists. J. Neurosci. 22 10377-10387.

Liu, F., Placzek, M., 2014. Axon guidance effects of classical morphogens Shh and BMP7 in the hypothalamo-pituitary system. Neurosci. Lett. 562, 108-113.

Massague, J., 2003. Integration of Smad and MAPK pathways: a link and a linker revisited. Genes Dev. 17, 2993-2997. 
McCabe, B.D., Marques, G., Haghighi, A.P., Fetter, R.D., Crotty, M.L., Haerry, T.E., Goodman, C.S., O'Connor, M.B., 2003. The BMP homolog Gbb provides a retrograde signal that regulates synaptic growth at the Drosophila neuromuscular junction. Neuron 39, 241-254.

Moustakas, A., Heldin, C.H., 2009. The regulation of TGFbeta signal transduction. Development 136, 3699-3714.

Ortega, J.A., Alcantara, S., 2010. BDNF/MAPK/ERK-induced BMP7 expression in the developing cerebral cortex induces premature radial glia differentiation and impairs neuronal migration. Cereb. Cortex 20, 2132-2144.

Tilleman, H., Hakim, V., Novikov, O., Liser, K., Nashelsky, L., Di Salvio, M.,

Krauthammer, M., Scheffner, O., Maor, I., Mayseless, O., Meir, I., Kayam, G. Sela-Donenfeld, D., Simeone, A., Brodski, C., 2010. Bmp5/7 in concert with the mid-hindbrain organizer control development of noradrenergic locus coeruleus neurons. Mol. Cell Neurosci. 45, 1-11.

Tsai, M.J., Pan, H.A., Liou, D.Y., Weng, C.F., Hoffer, B.J., Cheng, H., 2010. Adenoviral gene transfer of bone morphogenetic protein-7 enhances functional recovery after sciatic nerve injury in rats. Gene Ther. 17, 1214-1224.

Ueki, T., Tanaka, M., Yamashita, K., Mikawa, S., Qiu, Z.F., Maragakis, N.J., Hevner, R.F., Miura, N., Sugimura, H., Sato, K., 2003. A novel secretory factor,

neurogenesin-1, provides neurogenic environmental cues for neural stem cells in the adult hippocampus. J. Neurosci. 23, 11732-11740.

Yanagisawa, M., Takizawa, T., Ochiai, W., Uemura, A., Nakashima, K., Taga, T., 2001

Fate alteration of neuroepithelial cells from neurogenesis to astrocytogenesis by bone morphogenetic proteins. Neurosci. Res. 41, 391-396. 